\title{
混晶 $\mathrm{GaAs}_{1-x} \mathbf{P}_{x}(\mathrm{Te})$ 深中心的电声耦 \\ 合和光电特性 ${ }^{*}$
}

\author{
周必忠 林东海 黄景昭 陈世帛 \\ (嗄门大学物理系,厦门 361005)
}

关键词、 $\mathrm{GaAs}_{1-x} \mathrm{P}_{x}(\mathrm{Te})$ 、深中心、电声耦合

\section{一、引 言}

近年来, $\mathrm{III}-\mathrm{V}$ 族化合物混晶半导体中施主杂质(如 $\mathrm{Al}_{x} \mathrm{Ga}_{1_{-x}} \mathrm{As}$ 中 $\mathrm{Ge} 、 \mathrm{Si} 、 \mathrm{Sn}$ 和 $\mathrm{GaAs}_{1-x}$ $P_{x}$ 中 $S 、 S e 、 T e$ 等) 形成的深中心 ( $D X$ 中心) 的某些奇特行为引起人们广泛关注. 虽然这些 材料的基质和杂质的类型不同, 但深中心的特性相似, 例如它们在禁带中引人一个浅能级和个或几个深能级, 深能级的浓度很大 (可接近掺杂施主浓度); 深能级的光离化能显著大于热离 化能; 在低温, 电子俘获截面很小, 出现持续光电导或光电容 (PPC) 现象等。这些现象的产 生原因和缺陷本质目前仍有不同看法, 有待深入研究. 鉴于 $\mathrm{GaAs}_{s_{1-x}} \mathrm{P}_{x}(\mathrm{Te})$ 是一种重要光电 子材料, 该材料中施主深中心 (DX 中心) 是一种主要无辐射中心, 对材料、器件的特性有重要 影响; 迄今, 对 GaAsP(Te) 中 DX 中心的研究还较少. 研究发现: GaAsP(Te) 中存在多种 深中心 (如 A 中心和 $\mathrm{B}$ 中心等), 对 $\mathrm{A}$ 中心已有一些研究报道, 认为是 DX 中心, B 中心尚未见 到详细的研究报道. 本文报道用电学方法和光学方法系统研究 $\mathrm{GaAsP}(\mathrm{Te})$ 深中心(侧重 $\mathrm{B}$ 中 心)特性取得的有意义的结果. 用深能级瞬态谱 (DLTS) 测量 A 中心和 B 中心的热激活能, 研究了混晶无序引起的能级展宽和非指数性电容瞬态; 用光电容定态谱、瞬态谱和恒温光瞬态 谱 (OITS) 研究其光电性质, 在多种温度下测量了 B 中心的光离化截面谱, 确定光离化能, 研 究其声子展宽现象; 并且研究了电声耦合作用和晶格弛豫现象, 计算了晶格就像能, 给出位形 坐标图, 较好描述其特性.

\section{二、实验方法、结果及分析}

用深能级瞬态谱 (DLTS)、光电容定态谱、瞬态谱及 OITS 谱研究 $\mathrm{GaAs}_{1_{-x}} \mathrm{P}_{x}(\mathrm{Te}$ ) 深中 心的特性. 测量样品为 $\mathrm{GaAs}_{1_{-x}} \mathrm{P}_{x}(\mathrm{Te}) \mathrm{p}^{+} \mathrm{n}$ 结. 该样品以 $\mathrm{GaAs}$ 单晶为祄底, 用 MOCVD 法 生长掺 $T e$ 的 $n$ 型 $G a A s_{1-x} P_{x}$ 层, 后扩 $Z n$ 制成 $p^{+} n$ 结. 混晶组分用 $E L$ 谱结合带 $X$ 射线微区 分析的 SEM 测定. 测量主要结果及分析概括如下:

1. DLTS 谱和深能级展宽我们用高分辨深能级瞬态谱仪 (HDDLTS), 在标准的测 试条件下, 测量了多种 $\mathrm{GaAs}_{0.6} \mathrm{P}_{0.4}(\mathrm{Te})$ 样品的 DLTS 谱, 检测到两种较普遍存在的深 中心 ( $A$ 中心和 $B$ 中心). 典型结果作 Arrhenius 图, 如图 1 表示. 求得 $A$ 中心和 $B$ 中心的表观热

本文 1990 年 6 月 4 日收到.

* 国家自然科学蕉金和福建省科学基金资助项目. 


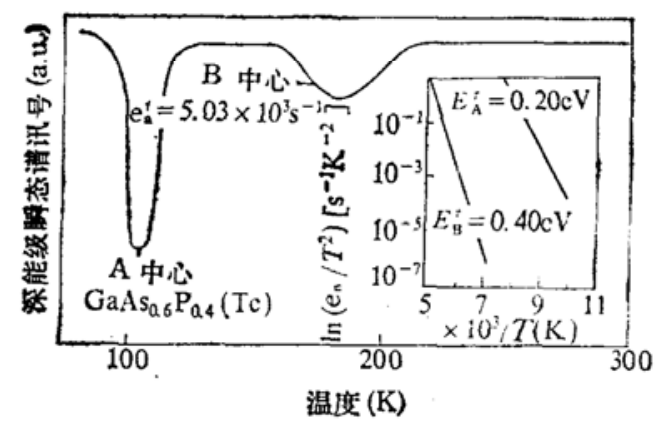

图 1 GaAs $0.6 \mathrm{P}_{0.4}(\mathrm{Te})$ DLTS 谱和 Arrhenius 图

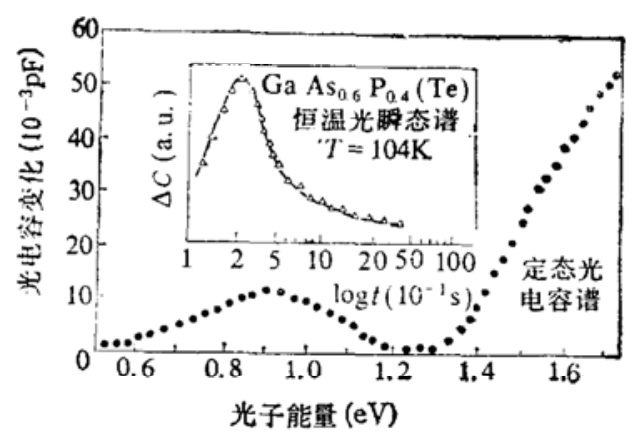

图 $2 \mathrm{GaAs}_{0.6} \mathrm{P}_{0.4}(\mathrm{Te})$ 定态电容谱及 OITS 毇

激活能分别为 $E_{\mathrm{A}}^{t}=0.20 \mathrm{eV}$ 和 $E_{\mathrm{B}}^{t}=0.40 \mathrm{eV}$. 发现 $\mathrm{B}$ 中心有明显的非指数电容瞬态和能级 展宽现象, 认为是由于混晶无序效应所致. 混晶中施主 $\mathrm{Te}$ 周围 $\mathrm{V}$ 族元素 $\mathrm{As}$ 和 $\mathrm{P}$ 原子的无 序分布, 引起混晶组分局部涨落, 造成杂质能级激活能偏离平均值, 围绕组分宏观平均值的涨 落, 产生能级展宽或多态组合, 造成能级上载流子发射的瞬态电容的非指数性.

2. 光电容定态谱、瞬态谱和光离化截面谱我们对相同样品测量了定态光电容谱, 典型 结桨如图 2 所示, 表明该样品存在两个对应 A 中心和 B 中心的深能级, 其光离化能精确数值由 瞬态光电容测量, 用光离化截面理论拟合确定, 分别为 $E_{\mathrm{A}}^{o}=0.60 \mathrm{eV}$ 和 $E_{\mathrm{B}}^{o}=1.31 \mathrm{eV}$. 由图 2 可见, 对于能量 $h v>1.2 \mathrm{eV}$ 的光激发, 光电容响应只是 $\mathrm{B}$ 中心上电子和空穴发射的贡献. 可以简便地用初始斜率法 ${ }^{[1]}$ 确定 $\mathrm{B}$ 中心的光漓化截面谱. 在恒定低温下, 测量光电容瞬态曲 线,求得其初始斜率:

$$
S^{0}(h \nu)=\left.\frac{d[\Delta C(t)]}{d t}\right|_{l=0}=\frac{N_{T} C_{0}}{2 N_{D}} e_{n}^{0}(h \nu),
$$

式中 $C_{0}$ 为没有光照样品电容, $N_{T}$ 和 $N_{D}$ 分别表示深能级和浅能级浓度, $\mathrm{e}_{n}^{0}(h v)$ 表示电子光 发射率, 满足:

$$
\mathrm{e}_{n}^{0}(h v)=\Phi \sigma_{n}^{0}(h v),
$$

因光电容瞬态测量过程光强 $\Phi$ 经等量子校对保持恒定, 故光离化截面 $\sigma_{\mathrm{n}}^{0}(h v)$ 和初始斜率成比 例, 因此由初始斜率测定可得到光离化截面谱. 用此方法较准确测量了 B 中心的光离化截面 谱,典型结果如图 3 表示.

设想 B 中心为定域化的深中心, 其局域势为短程势, 可用 $\delta$ 函数势模型(Lucovski 模型) ${ }^{[2]}$ 描述其光离化截面谱, 表示为

$$
\sigma_{\mathrm{n}}^{0}(h v)=\frac{16 \pi}{3} \frac{e^{2} \hbar}{\varepsilon_{0} n_{r} m_{c}^{*}}\left(E_{\mathrm{n}}^{0}\right)^{\frac{1}{2}}\left(h v-E_{\mathrm{n}}^{0}\right)^{3 / 2} /(h v)^{3},
$$

式中 $\theta_{0}$ 为真空介电常数, $n_{r}=\sqrt{\theta_{r}}, n_{r}$ 为半导体折射率, $\theta_{r}$ 为半导体介电常数, $m^{*}$ 为有效质 量, $C$ 为光速, $E_{\mathrm{n}}^{0}$ 为深中心的光离化能. 我们用此模型可较好拟合实测光离化截面谱, 得到 $\mathrm{B}$ 中心的光离化能 $E_{\mathrm{B}}^{o}(1.31 \mathrm{eV})$, 显著大于热激活能 $E_{\mathrm{b}}^{t}(0.40 \mathrm{eV})$.

3. OITS 谱 为了验证上:述结论的正确性, 我们研究了相闰样品的 OITS 谱 ${ }^{[3]}$. 用一 组取样时间 $t_{i}$ 和 $k t_{i}(k>1)$ 实时处理光电容䀦态曲线, 取信号:

$$
S(t, \sqrt{k}, T)=\left[\Delta C\left(k t_{i}\right)-\Delta C\left(t_{i}\right)\right] / \ln (k)
$$

作 $S\left(t_{i}, \sqrt{ } \bar{k}, T\right)$ 与 $t_{i}$ 的关系曲线, 即可得到 OITS 谱. 如果在时间轴上出现 $n$ 个谱峰, 则 
相应有 $\boldsymbol{n}$ 个能级. 我们用计算机拟合光电容瞬态曲线, 结果如图 2 中插图所示, OITS 谱只 出现一个谱峰, 证明光子能量 $h \nu>1.2 \mathrm{eV}$ 的光激发引起的光电容响应为单能级 (B 中心) 的 贡献.

4. 低温持续光电导或持久光电容效应 我们在低温观察到 $\mathrm{GaAs}_{0.6} \mathrm{P}_{0.4}(\mathrm{Te}) \mathrm{B}$ 中心的 PPC 现象. 在温度近 $90 \mathrm{~K}$, 用能量 $h v>1.3 \mathrm{eV}$ 的单色光照射样品, 使 $\mathrm{B}$ 中心激发载流子, 达 到光电容稳定值, 随后样品加零偏注人电子, 在反偏下, 再用 $h v>1.3 \mathrm{eV}$ 的单色光照射样品, 结果发现没有明显的光电容响应, 表明 $\mathrm{B}$ 中心的低温电子俘获截面很小. 如果将样品温度升 高到 $100 \mathrm{~K}$ 以上, 重复上述过程, 则可以观察到明显的光电容响应, 表现 $\mathrm{B}$ 中心的俘获截面随 温度升高而显著增大. 这种在低温电子俘获截面很小, 具有强烈温度依赖关系的特性正反映 了 DX 中心的基本虽性.

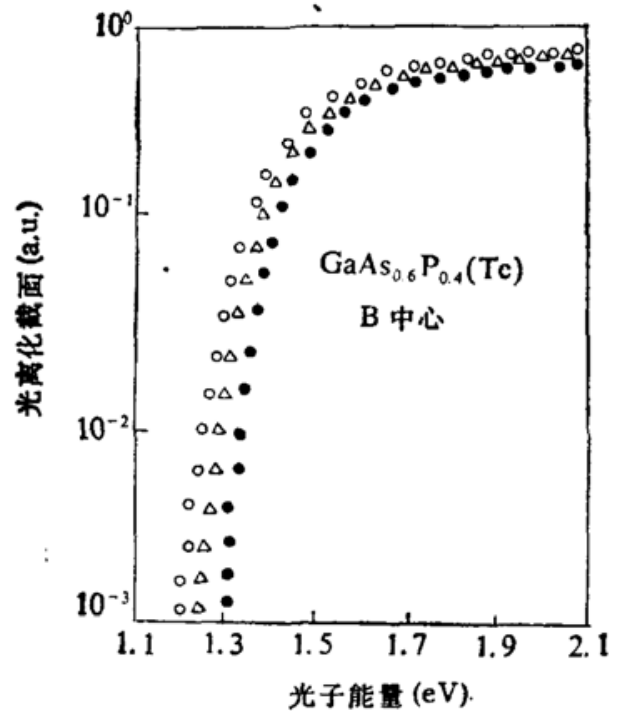

图 $3 \mathrm{GaAs}_{0.0} \mathrm{P}_{0.4}(\mathrm{Te})$ B 中心光离化截面谱声子展宽 $\bullet-104 \mathrm{~K} ; \Delta-1+8 \mathrm{~K} ; 0-197 \mathrm{~K}$

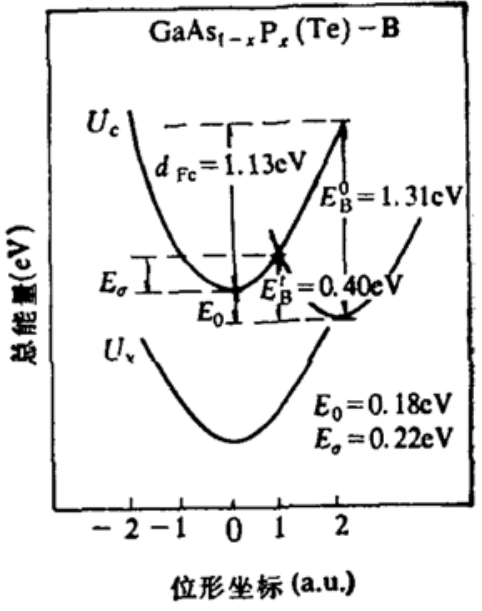

图 $4 \mathrm{GaAs}_{0.0} \mathrm{P}_{0.4}(\mathrm{Te})$ B 中心位形坐标图

5. B 中心的电声耦合和晶格弛像混晶半导体深中心的显著特点在于深中心的局域电 子态与周围晶格原子之间存在强烈电声耦合作用, 因此当局域态电子发生跃迁时, 必然晶格原 子的平衡位置会发生移动, 产生晶格弛豫, 并且导致深中心的光离化谱随温度变化而移动的声 子展宽现象. 迄今, $\mathrm{GaAs}_{0.6} \mathrm{P}_{0.4}(\mathrm{Te}) \mathrm{B}$ 中心光离化截面 $\left(\sigma_{\mathrm{B}}^{o}\right)$ 谱的声子展宽现象尚末见有研 究报道. 我们作了较仔细研究, 在多种温度下, 测量了 $\sigma_{\mathrm{B}}^{\circ}(h v)$ 温度关系(如图 3), 发现: 随温 度升高 $\omega_{\mathrm{R}}^{o}$ 谱移向低能端, 有明显的声子展宽.

如果我们考虑 $\mathrm{GaAs}_{1_{-x}} \mathrm{P}_{x}(\mathrm{Te}) \mathrm{B}$ 中心存在强电声耦合. 根据 Jaros 理论 ${ }^{[4]}$, 将 B 中心的 光离化截面用下式描述:

$$
\begin{aligned}
\sigma_{\mathrm{B}}^{o}(h v)= & \frac{1}{h v} \int_{0}^{\infty} d E \rho(E) x\left|\frac{(1 \pm \eta) E^{\frac{1}{2}}}{E_{\mathrm{B}}^{o}+E}+\frac{(1 \mp \eta) E_{F}^{\frac{1}{2}}}{E_{\mathrm{B}}^{o}-E-\frac{1}{2}\left(E_{\mathrm{g}}+E_{\mathrm{p}}\right)}\right|^{2} \\
& \cdot \exp \left\{-\frac{\left[h v-\left(E_{\mathrm{B}}^{o}+E\right)\right]}{4 k T d_{\mathrm{Fc}}}\right\},
\end{aligned}
$$

式中 $\rho(E)$ 为态密度, $E$ 为电子态能量, $d_{\mathrm{Fc}}$ 为晶格弛豫能, $E_{\mathrm{p}}$ 为 Penn 间隙 ${ }^{\left[{ }^{5}\right.}, \eta=\exp (-2 E$ / 
$E_{\mathrm{p}}$ ), 符号“ \pm ”对应类价带或类导带的缺陷态, 则此现象可以得到理论解释。

我们还分析了 $\mathrm{B}$ 中心的晶格弛豫现象. 根据昆格弛豫理论 ${ }^{[6]}$, 计算了 $\mathrm{B}$ 中心的晶格弛豫 能。利用晶格驰豫能 $d_{\mathrm{fo}_{\mathrm{o}}}$ 和深中心束缚能 $E_{0}$ 之间的关系:

$$
\begin{aligned}
& d_{\mathrm{P}_{\mathrm{c}}}=E_{\mathrm{B}}^{o}-E_{0}, \\
& \bar{E}_{0}=E_{\mathrm{B}}^{t}-E_{\sigma},
\end{aligned}
$$

$E_{\sigma}$ 为深中心的俘获势垒. 我们引用 $\mathrm{Kaniewska}{ }^{[7]}$ 最近对 $\mathrm{GaAs}_{0.6} \mathrm{P}_{0.4}(\mathrm{Te}) \mathrm{B}$ 中心 的 $E_{0}$ 精确 测量结果, 算出了 $E_{0}=0.18 \mathrm{eV}$, 根据我们实验结果 $E_{\mathrm{B}}^{o}=1.31 \mathrm{eV}$, 可算出 $d_{\mathrm{Fc}}=1.13 \mathrm{eV}$. 通常可以认为: 如果 $d_{\mathrm{P}_{\mathrm{c}}}>E_{0}$, 则该深中心有大晶格弛豫. 由我们研究结果表明 $\mathrm{GaAs}_{0.6} \mathrm{P}_{0.4}$ ( Te) $\mathrm{B}$ 中心的 $d_{\mathrm{F}_{\mathrm{c}}}$ 比 $E_{0}$ 大得多, 因此可认为具有大晶格驰豫. 根据上述结果, 我们作出 $\mathrm{GaAs}_{0.6} \mathrm{P}_{0.4}(\mathrm{Te}) \mathrm{B}$ 中心的外交叉型的位形坐标图(如图 4 表示), 可以较好地描述 B 中心的特 性.

\section{三、结 束 语}

通过用电学方法和光学方法对 $\mathrm{GaAs}_{1_{-x}} \mathrm{P}_{x}$ : Te 中杂质缺陷的综合研究, 结果发现: 混昆 $\mathrm{GaAs}_{1_{1-x}} \mathrm{P}_{x}$ 掺人施主杂质 ( $\mathrm{Te}$ ) 后, 不仅引入浅施主能级, 而且产生深施主中心(如 $\mathrm{A}$ 中心和 $\mathrm{B}$ 中心); $\mathrm{B}$ 中心的光离化能显著大于热激活能; 有明显的混晶无序引起的能级展宽和非指数性 电容瞬态; 其光离化截面谱随温度改变而移动出现声子展宽现象; 在低温 ( $90 \mathrm{~K}$ ) 存在持续光电 导或持久光电容效应等. 表明 $\mathrm{B}$ 中心具有 $\mathrm{DX}$ 中心的一些基本属性, 并且具有强的电声耦合 作用和大的晶格弛豫, 说明 B 中心是一和有效的无辐射复合中心, 对 GaAsP 材料和器件的特 性(如发光效率及稳定性) 可能产生深刻影响. 对于这类缺陷的结构和复杂的特性, 我们将进 一步深人探讨, 另作报道.

\section{参考文献}

[1] Wang, Z.G. et al., J. Phys. C: Solid State Phys., 17 (1984), 259.

[2] Lucovski, G., Solid Szate Electronics, 3 (1965), 299.

[3] Morante, J.R. et al., J. Appl. Phyc., 60 (1986), 1661.

[4] Jaros, M., Phys. Rev., Bl6 (1977), 3694.

[5] Penn. D. R., Phys. Rev., 128(1962), 2093.

（6]黄昆,物理学进展, 1(1980), 20 .

[7] Kaniewsika, M. and Kaniewski, J., J. Appl. Phys., 63 (1988), 1086. 